\title{
Different functions of AKT1 and AKT2 in molecular pathways, cell migration and metabolism in colon cancer cells
}

\author{
SARA HÄGGBLAD SAHLBERG ${ }^{1}$, ANJA C. MORTENSEN ${ }^{1}$, JAKOB HAGLÖF ${ }^{2}$, \\ MIKAEL K.R. ENGSKOG ${ }^{2}$, TORBJÖRN ARVIDSSON ${ }^{2}$, CURT PETTERSSON ${ }^{2}$, \\ BENGT GLIMELIUS $^{1}$, BO STENERLÖW ${ }^{1}$ and MARIKA NESTOR ${ }^{1,3}$
}

\begin{abstract}
${ }^{1}$ Department of Immunology, Genetics and Pathology, The Rudbeck Laboratory, Uppsala University, SE-751 85 Uppsala;
${ }^{2}$ Division of Analytical Pharmaceutical Chemistry, Department of Medicinal Chemistry, Uppsala University, SE-751 23 Uppsala; ${ }^{3}$ Science for Life Laboratory, Uppsala University, SE-751 85 Uppsala, Sweden
\end{abstract}

Received August 3, 2016; Accepted October 12, 2016

DOI: $10.3892 /$ ijo.2016.3771

\begin{abstract}
AKT is a central protein in many cellular pathways such as cell survival, proliferation, glucose uptake, metabolism, angiogenesis, as well as radiation and drug response. The three isoforms of AKT (AKT1, AKT2 and AKT3) are proposed to have different physiological functions, properties and expression patterns in a cell type-dependent manner. As of yet, not much is known about the influence of the different AKT isoforms in the genome and their effects in the metabolism of colorectal cancer cells. In the present study, DLD-1 isogenic $A K T 1, A K T 2$ and $A K T 1 / 2$ knockout colon cancer cell lines were used as a model system in conjunction with the parental cell line in order to further elucidate the differences between the AKT isoforms and how they are involved in various cellular pathways. This was done using genome wide expression analyses, metabolic profiling and cell migration assays. In conclusion, downregulation of genes in the cell adhesion, extracellular matrix and Notch-pathways and upregulation of apoptosis and metastasis inhibitory genes in the p53-pathway, confirm that the knockout of both $A K T 1$ and $A K T 2$ will attenuate metastasis and tumor cell growth. This was verified with a reduction in migration rate in the $A K T 1 \mathrm{KO}$ and $A K T 2 \mathrm{KO}$ and most explicitly in the $A K T 1 / 2 \mathrm{KO}$. Furthermore, the knockout of $A K T 1, A K T 2$ or both, resulted in a reduction in lactate and alanine, suggesting that the metabolism of carbohydrates and glutathione was impaired. This was further verified in gene expression analyses, showing downregulation of genes involved in glucose metabolism. Additionally, both $A K T 1 \mathrm{KO}$ and $A K T 2 \mathrm{KO}$ demonstrated an impaired fatty acid
\end{abstract}

Correspondence to: Dr Marika Nestor, Department of Immunology, Genetics and Pathology, The Rudbeck Laboratory, Uppsala University, SE-751 85 Uppsala, Sweden

E-mail: marika.nestor@igp.uu.se

Key words: microarray, metabolism, cell migration AKT1, AKT2, AKT, PKB, gene expression, colon cancer, DLD-1, metabolic profiling, CD44, CD133 metabolism. However, genes were upregulated in the Wnt and cell proliferation pathways, which could oppose this effect. AKT inhibition should therefore be combined with other effectors to attain the best effect.

\section{Introduction}

Cancer progression involves multiple pathways and steps leading to cell growth and proliferation. New therapeutic strategies targeting cell growth and proliferation include several AKT inhibitors, even though little knowledge of their effects in different cell-types or on the genome exists (1). In order to predict their effects, as well as possible side-effects, it is important to understand how AKT, particularly the different AKT isoforms, take part in the different cellular pathways.

AKT is the central protein of a major cell signaling system involved in cell survival, metastasis, drug resistance, metabolism and radiation resistance (2). While inhibition of AKT can result in inhibition of important pathways for cell-survival, it may also lead to stimulation of other downstream proteins such as receptor tyrosine kinases (e.g. HER3, IGF-1R and insulin receptor), enabling the cancer cells to survive (3). There are three AKT isoforms, AKT1, AKT2 and AKT3, which have high homology but are expressed from three separate genes. Their expression is tissue-dependent and they are believed to have different functions. Knockout mouse studies have shown AKT1 to be essential for cell survival, AKT2 to have a more prevalent role in glucose homeostasis, while AKT3 is believed to be involved in brain development (4). Overexpression of the different AKT isoforms has been seen in several types of cancer. An elevated expression of AKT1 has been observed in GIST, thyroid and breast cancer, whereas high AKT2 expression has been seen in glioma, colorectal, hepatocellular, ovarian and pancreatic cancer, and AKT3 overexpression has been observed in breast and prostate cancer $(2,5)$. In several studies, AKT signaling is associated with enhanced cell motility and invasion (6). However, recent in-depth studies on the AKT isoforms using siRNA, isoform specific inhibitors, AKT isoform expressing vectors and AKT isoform gene knockouts, have shown that AKT1 and AKT2 might play different roles in this pathway depending on the cell type (6). 
Cancer cells generally grow faster than normal cells and often display an increase in glucose uptake and lactate production (7). AKT plays a role in cellular metabolism through its association with glucose uptake via the glucose transporters (GLUT), as well as the conversion of stored glycogen back to glucose. There are several studies showing that AKT1 and AKT2 have different roles in glucose homeostasis (8). AKT2 silencing in mice was shown to cause an impaired glucose uptake by fat and muscle cells (9). Furthermore, in vitro studies have demonstrated that $A K T 2$ silencing causes inhibition of insulin induced GLUT4 translocation to the plasma membrane. GLUT4 promotes an increase of glucose in the cells when situated in the plasma membrane (10). It has also been proposed that glycolysis can result in formation of pyruvate and NADPH, which can reduce reactive oxygen species and thereby reduces oxidative stress (11).

Only a few studies have evaluated the effects of the different AKT isoforms in colorectal cancer. We have previously shown that both AKT1 and AKT2 interact with the DNA-repair protein DNA-PKcs and that disruption of these increases radiation sensitivity and influences the expression of cancer stem cell markers CD44 and CD133 $(12,13)$. While the focus of previous studies has been on a few specific pathways, the present study aimed to perform a genome wide expression profile in $A K T$ isoform knockout colon cancer cells. Additionally, metabolomic and cell migration studies could further elucidate the function of the AKT isoforms in colorectal cancer. This may help to improve treatment by assessing new targets for combination therapy or finding biomarkers for prediction of treatment response.

\section{Materials and methods}

Cell culture. The colon cancer isogenic DLD-1 X-MAN ${ }^{\mathrm{TM}}$ cell lines were obtained from Horizon Discovery Ltd., (Cambridge, UK) with the different AKT isoforms genetically knocked out, cat. no. HD-R00-001, HD-R00-002 and HD-R00-003. The cells were cultured in $75-\mathrm{cm}^{2}$ culture flasks (Nunclon surface; Nunc, Roskilde, Denmark) in McCoy's 5A medium (Flow Laboratories, Irvine, UK) with $10 \%$ fetal bovine serum (FBS; Sigma-Aldrich, St. Louis, MO, USA), $2 \mathrm{mM}$ L-glutamine, $100 \mathrm{IU} / \mathrm{ml}$ penicillin and $10 \mu \mathrm{g} / \mathrm{ml}$ streptomycin (Biochrom GmbH, Berlin, Germany). The cells were cultured in a humidified incubator with $5 \% \mathrm{CO}_{2}$ at $37^{\circ} \mathrm{C}$ and trypsinized with trypsin-EDTA, $0.25 \%$ trypsin, $0.02 \%$ EDTA (Biochrom GmbH).

Microarray expression analysis. Two separate passages of DLD-1 parental, AKT1 KO, AKT2 KO and AKT1/2 KO cells were cultured to $70 \%$ confluence and RNA was extracted (RNeasy MiniPrep; Qiagen, Valencia, CA, USA). The RNA concentration was measured with ND-1000 spectrophotometer (NanoDrop Technologies, Wilmington, DE, USA) and RNA quality was evaluated using the Agilent 2100 Bioanalyzer system (Agilent Technologies, Inc., Palo Alto, CA, USA). A total of $250 \mathrm{ng}$ of total RNA from each sample was used to generate amplified and biotinylated sense-strand cDNA from the entire expressed genome according to the GeneChip ${ }^{\circledR}$ WT PLUS reagent kit user manual (P/N 703174 Rev.1; Affymetrix, Inc., Santa Clara, CA, USA). GeneChip ${ }^{\circledR}$ HTA arrays
(GeneChip ${ }^{\circledR}$ Human Transcriptome array 2.0) were hybridized for $16 \mathrm{~h}$ in a $45^{\circ} \mathrm{C}$ incubator, rotated at $60 \mathrm{rpm}$. According to the GeneChip ${ }^{\circledR}$ expression, Wash, Stain and Scan Manual (P/N 702731 Rev.3; Affymetrix) the arrays were then washed and stained using the Fluidics Station 450 and finally scanned using the GeneChip ${ }^{\circledR}$ Scanner $30007 \mathrm{G}$.

Microarray data analysis. The raw data was normalized in the free software Expression Console provided by Affymetrix (http://www.affymetrix. com) using the robust multi-array average (RMA) method first suggested by Li and Wong in 2001 (14). Subsequent analysis of the gene expression data was carried out in the freely available statistical computing language R (http://www.r-project.org) using packages available from the Bioconductor project (www.bioconductor.org). In order to search for the differentially expressed genes between parental and the $A K T \mathrm{KO}$, an empirical Bayes moderated t-test was applied, using the 'limma' package (15). To address the problem with multiple testing, P-values were adjusted using the method of Benjamini and Hochberg (16).

Pathway analysis. DAVID Bioinformatic resources 6.7 software was used to functionally classify and cluster the genes with an altered expression and identify the most significantly altered pathways, networks and metabolism processes that the genes were involved in. Only genes with a 1.5-fold change and with a $\mathrm{P}<0.05$ were used in the evaluation. To rank and calculate P-values for the different pathways and processes normalized data from all four cell lines was compared. The P-value represents the probability for a particular mapping of an experiment to a process arisen by chance, considering the number of genes in the experiment versus the number of genes in the map process.

Scratch wound migration assay. In this study, DLD-1 isogenic $A K T 1, A K T 2$ and $A K T 1 / 2$ knockout colon cancer cell lines were used as a model system together with the parental cell line in order to further elucidate the differences between the AKT isoforms and how they are involved in various cellular pathways. Many of these pathways are thought to be involved in cellular migration. Thus, the migratory activity of isogenic $A K T 1, A K T 2$ and $A K T 1 / 2$ knockout colon cancer cell lines were studied together with the parental cells, using the scratch wound migration assay (17). The migration rate was monitored at two different settings, complete medium and after starvation plus addition of EGF. DLD-1 parental and $A K T$ knockout cells were seeded in 6-well plates in McCoy's 5A medium (Flow Laboratories). After 24 h the cells were washed three times with $2 \% \mathrm{FBS} / \mathrm{McCoy}$ 's medium and incubated in starvation medium, 2\% FBS/McCoy's, for another $24 \mathrm{~h}$. The cells were wounded with a sterile plastic pipette and rinsed three times to remove cellular debris. Subsequently, complete medium, 10\% FBS/McCoy's, plus 15 ng human recombinant EGF (Chemicon) was added. Migration was monitored using a Nikon 700D digital camera mounted on an inverted microscope at time 0-24 h after wounding and analyzed using ImageJ software. Statistical analyses were performed using GraphPad Prism version 6 for Windows (GraphPad Software, Inc., La Jolla, CA, USA). The differences in uptake between the $A K T \mathrm{KO}$ cell lines compared to the parental cell line were 
evaluated using two-way ANOVA with multiple comparison test and were considered statistically significant at $\mathrm{P}<0.05$.

Cell harvesting for metabolic profiling. Cells were cultured in $12-\mathrm{cm}$ petri dishes and harvested at $\sim 75 \%$ confluence. All cell sample harvesting was performed on ice. Growth medium was removed and cells were rapidly washed three times with cold Dulbecco's phosphate-buffered saline (PBS; Nordic Biolabs AB, Täby, Sweden) followed by detachment of cells using a rubber-tipped cell scraper (Sarstedt). The detached cells were collected in cold Milli-Q water $(3.5 \mathrm{ml})$, transferred to polypropylene tubes and snap-frozen in liquid $\mathrm{N}_{2}$ followed by thawing at $37^{\circ} \mathrm{C}$ for $10 \mathrm{~min}$. The freeze-thaw cycle was then repeated once with subsequent sonication on ice for $30 \mathrm{sec}$. Protein concentrations were measured in duplicates using the spectrophotometric assay with Bradford reagent (Sigma-Aldrich) and using bovine serum albumin (BSA; Sigma-Aldrich) for reference samples, all according to the manufacturer's instructions. Samples were stored at $-80^{\circ} \mathrm{C}$ until metabolite extraction. For each of the four cell lines, three technical replicates were harvested.

Extraction of polar metabolites. Quenched cells were thawed at room temperature and subjected to centrifugation $(10 \mathrm{~min}$, $2200 \mathrm{RCF}$ ) to remove precipitated cellular debris. The supernatant of the cell extracts was transferred to a fresh extraction tube, followed by addition of chloroform and methanol in the proportions 4:4:2.85 $\left(\mathrm{MeOH}: \mathrm{CHCl}_{3}: \mathrm{H}_{2} \mathrm{O}\right)$. The resulting two-phase system was gently mixed and left at $4^{\circ} \mathrm{C}$ for $30 \mathrm{~min}$ prior to centrifugation $\left(2200 \mathrm{RCF}, 20 \mathrm{~min}, 4^{\circ} \mathrm{C}\right)$. A fixed volume of the aqueous phase was transferred to a new tube and evaporated under $\mathrm{N}_{2}$ at $40^{\circ} \mathrm{C}$ until dry. The polar phase was subsequently reconstituted in $0.7 \mathrm{ml}$ phosphate-buffered $\mathrm{D}_{2} \mathrm{O}$ (150 mM, pD 7) containing 2,2-dimethyl-2-silapentane5-sulfonate sodium salt (DSS; $34 \mu \mathrm{M})$ as a chemical shift reference. For the analysis, one pooled quality control (QC) sample was also created by pooling an equal volume of cell extracts prior to metabolite extraction.

Metabolic profiling using NMR spectroscopy. NMR measurements were carried out at $298 \mathrm{~K}$ on a Bruker Avance $600 \mathrm{MHz}$ (Bruker BioSpin GmbH, Rheinstetten, Germany) equipped with a cryoprobe. For each sample, the 1D NOESYPR1D standard pulse sequence (-RD- $90^{\circ}-\mathrm{t}_{1}-90^{\circ}-\mathrm{t}_{\mathrm{m}}-90^{\circ}$-ACQ) was used. Each pulse had a $90^{\circ}$ pulse length; the total amount of FIDs recorded were 256 collected into $64 \mathrm{~K}$ data points which was zero-filled to $128 \mathrm{~K}$ data points. The spectra width was set to $7183.91 \mathrm{~Hz}$ giving a spectral acquisition time of $4.56 \mathrm{sec}$. The $\mathrm{t}_{1}$ and $\mathrm{t}_{\mathrm{m}}$ was set to $6 \mu \mathrm{sec}$, respectively, $180 \mathrm{msec}$ and relaxation delay (D1) was $3 \mathrm{sec}$, resulting in a total acquisition time of $33 \mathrm{~min}$. The 1D spectra were manually phased and baseline corrected and the ${ }^{1} \mathrm{H}$ chemical shifts were referenced to added DSS using the ACD/Labs (version 12.01; Advanced Chemistry Development, Inc., Toronto, ON, Canada). Each ${ }^{1} \mathrm{H}$ NMR spectrum, within a range of $0-10 \mathrm{ppm}$, was reduced to 873 bins of fixed width (0.01 ppm) excluding resonance regions for water $(\delta 5.15-4.67)$ and the internal standard (DSS, $80.00-0.65,1.77-1.72$ and 2.922.88). The signal intensity in each bin was integrated and data were imported, normalized to unit total intensity in Microsoft Excel (Microsoft Office 2007; Microsoft, Redmond, CA, USA). Assignments of NMR peaks were performed according to the
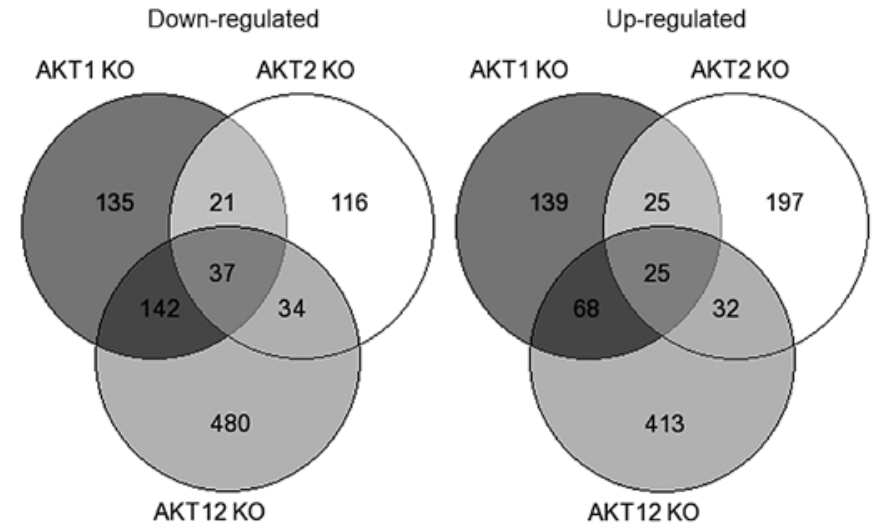

Figure 1. Venn diagram from gene expression analysis. A comparison of down-regulated and up-regulated genes in the $A K T$ isoform knockout cell lines.

metabolomics standards initiative (MSI) (18) by comparing with the Human Metabolome Database (v2.5) (19). The metabolites identified should be seen as putatively annotated compounds according to the MSI nomenclature.

Multivariate data analysis of metabolic profiling data. Multivariate data analysis was conducted in SIMCA-P+ (version 13.0; MKS Umetrics AB, Umeå, Sweden) computational software package. Data were Pareto scaling, as implemented in the SIMCA-P+ package, prior to further analysis. Unsupervised principal component analysis (PCA) and supervised orthogonal projection to latent structures discriminant analysis (OPLS-DA) was used for identification of key differences between parental cell lines and $A K T \mathrm{KO}$ cell lines (20).

\section{Results}

Gene expression assay. Comparing the different altered pathways in the $A K T 1 \mathrm{KO}, A K T 2 \mathrm{KO}$ and $A K T 1 / 2 \mathrm{KO}$ celllines, a number of signaling pathways were altered according to the functional annotation analysis, including cell adhesion molecules (CAMs), extracellular matrix (ECM)-receptor interaction, Notch, Wnt and p53 pathways (Figs. 1 and 2, and Table I).

These pathways were further analyzed to evaluate the importance of the different genes. There were also differences in the metabolic pathways such as the starch, sucrose, glucose pathway, the glutathione metabolism and fatty acid metabolism. Note that some of these genes are involved in more than one pathway (Fig. 3 and Table II).

In the cell adhesion molecule (CAM) pathway, several genes were downregulated in the $A K T 1 / 2 \mathrm{KO}$ cell line compared to parental; ALCAM,CD99, CDH1, CLDN1, CLDN2, HLA-DMA, ITGA9, NEO1, PTPRF, SDC1, MET, RAC2, KIT and TGFA. Genes that were upregulated include $I G F 1 R, P T K 2, F O X O 1$ and $P A L L D$. The AKT1 KO cells displayed an analogous pattern. The $A K T 2 \mathrm{KO}$ cells on the other hand, showed upregulation of several genes in this pathway; CADM1, CLDN1,ITGB8, NEO1, PVRL3, SDC4, PALLD.

In the ECM-receptor interaction pathway, several genes were altered in the $A K T 1 / 2 \mathrm{KO}$ cell line compared to parental 


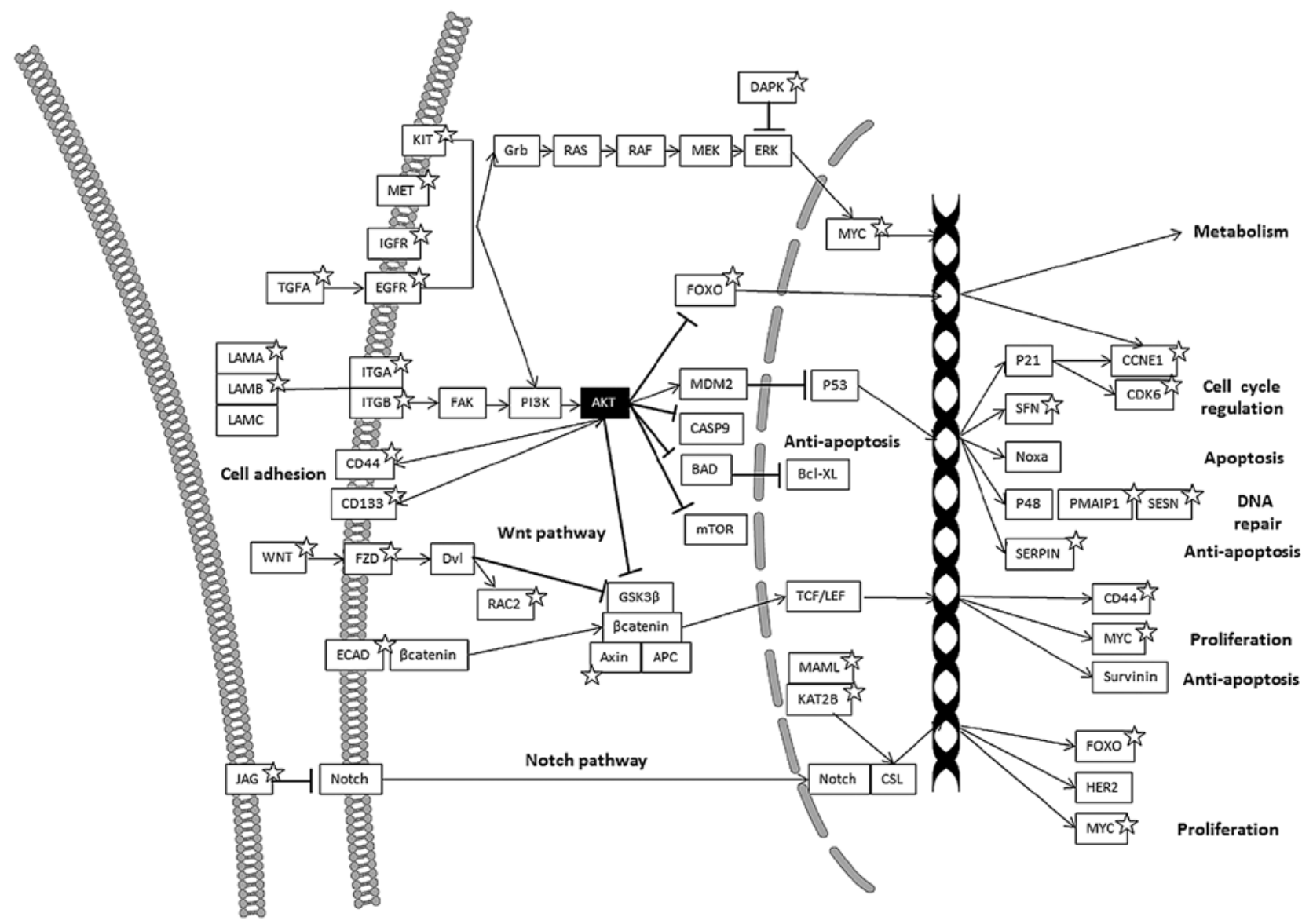

Figure 2. AKT signaling pathway map. AKT is involved in many different signaling pathways such as metabolism, cell cycle regulation, cell survival, DNA repair and proliferation. Arrows mean activation, thick lines mean inhibition and stars indicate genes with differences in expression levels between AKT isoform knockout cell lines and parental cells according to the gene expression analysis.
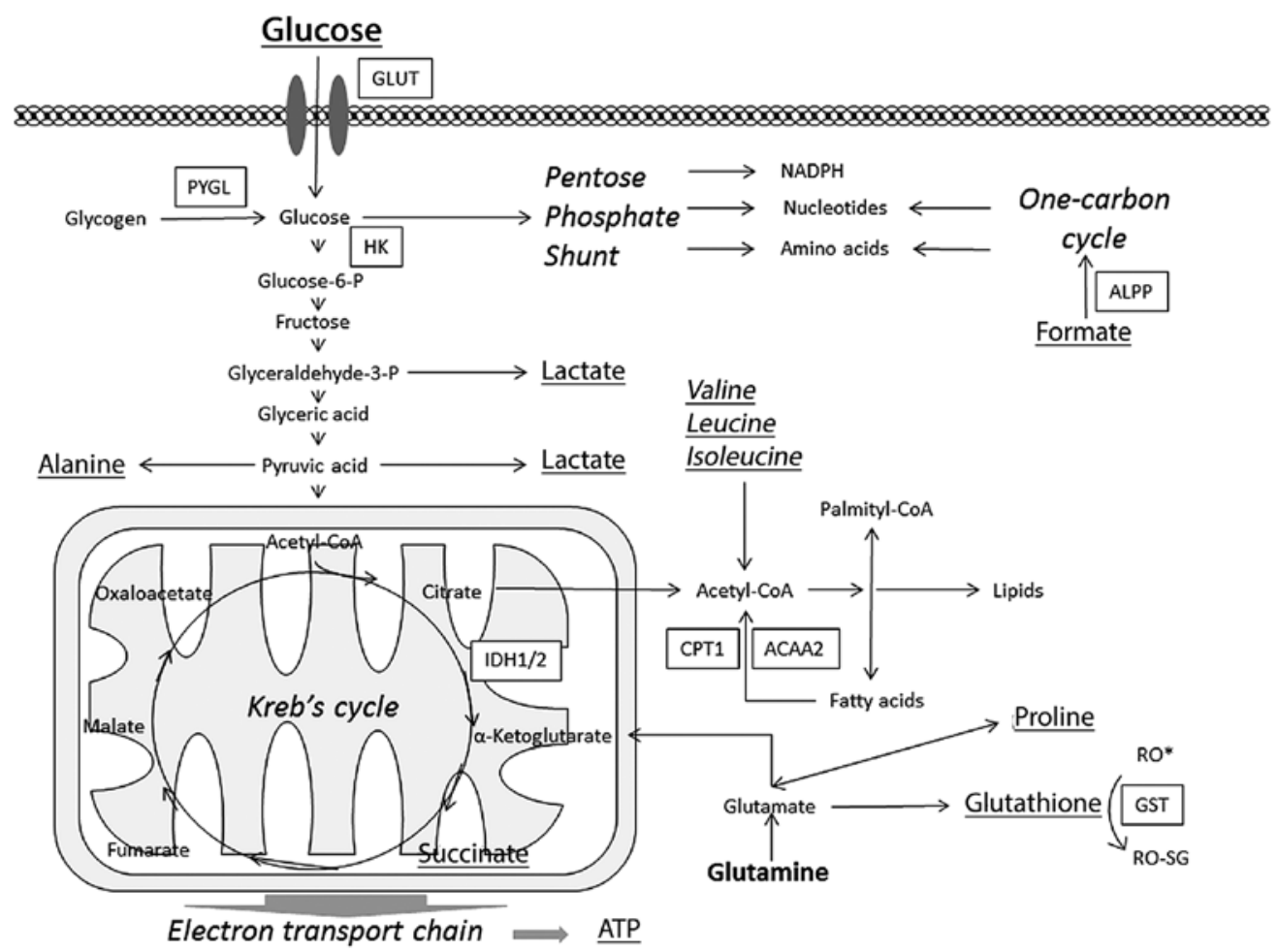

Figure 3. A simplified view of the metabolic pathways. A selection of genes with altered expression in the AKT knockout cell lines compared to parental cells according to the gene expression analysis are shown in boxes. Metabolites altered in the AKT knockout cells compared to parental cells according to the metabolome analysis are shown underlined. 
Table I. Functional annotation chart report of KEGG signaling pathways from DAVID using the downregulated, upregulated or both downregulated and upregulated gene set for each subgroup.

\begin{tabular}{lll}
\hline Pathways & P-value Genes
\end{tabular}

AKT1 KO vs. parental

Downregulated genes

Notch signaling pathway

Upregulated genes

ECM-receptor interaction

Focal adhesion

Pathways in cancer

Both upregulated and downregulated genes

ECM-receptor interaction

Focal adhesion

Pathways in cancer

Prostate cancer

Colorectal cancer

AKT2 KO vs. parental

Downregulated genes

N/A

Upregulated genes

Cell adhesion molecules (CAMs)

MAPK signaling pathway

Both upregulated and downregulated genes

Cell adhesion molecules (CAMs)

ECM-receptor interaction

$A K T 1 / 2 \mathrm{KO}$ vs. parental

Downregulated genes

Cell adhesion molecules (CAMs)

ECM-receptor interaction

Notch signaling pathway

Upregulated genes

p53 signaling pathway

Pathways in cancer

Upregulated and downregulated genes

ECM-receptor interaction

Pathways in cancer
5.27E-03

7.87E-02

7.97E-03

5.88E-02

3.93E-03

$6.22 \mathrm{E}-02$

1.25E-02 LAMA2, CD47, ITGA9, SDC1, CD44, TNC, ITGA1, ITGB5, ITGA2, ITGA3, LAMB1

CADM1, ITGB8, PVRL3, CLDN1, NEO1, SDC4

BDNF, CACNA2D1, RASGRF2, MAP3K14, FLNA, TGFB2

ITGA9, CADM1, ITGB8, PVRL3, CLDN1, CLDN2, NEO1, SDC4 ITGA9, CD44, ITGB8, TNC, SDC4

1.80E-02 ALCAM, ITGA9, SDC1, PTPRF, CLDN1, CLDN2, CD99, CDH1, NEO1, HLA-DMA

4.10E-02 LAMA2, CD47, ITGA9, SDC1, TNC, ITGA3, LAMB1

5.27E-02 DTX4, HES1, KAT2B, MAML2, JAG1

CCNE2, CCNE1, SERPINB5, CDK6, RRM2B, PMAIP1, SESN3

WNT16, RALBP1, ITGA2, FOXO1, CDK6, APPL1, FZD7, FZD6, CCNE2, IGF1R, CCNE1, PTK2, TCEB1

WNT16, PPARG, ARNT2, TGFB3, EGLN3, FOXO1, CDH1, KIT, AKT1, CCNE2, IGF1R, CCNE1, PTK2, RAC2, TGFA, LAMB1, RALBP1, MET, SKP2, ITGA2, CDK6, ITGA3, APPL1, FZD7, FZD6, DAPK1, LAMA2, TCEB1 
Table II. Functional annotation chart report of KEGG metabolic signaling pathways from DAVID using the downregulated, upregulated or both downregulated and upregulated gene set for each subgroup.

\begin{tabular}{lll}
\hline Pathways & P-value Genes
\end{tabular}

$A K T 1 \mathrm{KO}$ vs. parental

Downregulated genes

N/A

Upregulated genes

Folate biosynthesis

Both upregulated and downregulated genes

Folate biosynthesis

$A K T 2 \mathrm{KO}$ vs. parental

Downregulated genes

N/A

Upregulated genes

N/A

Both upregulated and downregulated genes

Arginine and proline metabolism

6.49E-02 SAT1, ALDH7A1, CKMT1A, CKMT1B, MAOB

$A K T 1 / 2 \mathrm{KO}$ vs. parental

Downregulated genes

Valine, leucine and isoleucine degradation
1.91E-03 ALDH6A1, ACADSB, MUT, HMGCS1, ALDH2, ABAT, ACAA1
$3.70 \mathrm{E}-03$
$9.10 \mathrm{E}-03$
$1.06 \mathrm{E}-02$
GSTM1, GSTM2, GSTM3, GSTM4, IDH2, IDH1, MGST1
GSTM1, GSTM2, GSTM3, GSTM4, UGT1A5, UGT2B10, MGST1
GSTM1, GSTM2, GSTM3, GSTM4, UGT1A5, UGT2B10, MGST1

Glutathione metabolism

Metabolism of xenobiotics by cytochrome P450

Drug metabolism

Ascorbate and aldarate metabolism

Propanoate metabolism

Pentose and glucuronate interconversions

Butanoate metabolism

Androgen and estrogen metabolism

Starch and sucrose metabolism

Amino sugar and nucleotide sugar metabolism

Steroid hormone biosynthesis

Retinol metabolism

Upregulated genes

N/A

Both upregulated and downregulated genes

Glutathione metabolism

Starch and sucrose metabolism

Valine, leucine and isoleucine degradation

Butanoate metabolism

Drug metabolism

Ascorbate and aldarate metabolism

Pentose and glucuronate interconversions

Propanoate metabolism
$1.36 \mathrm{E}-02$

$1.50 \mathrm{E}-02$

$1.59 \mathrm{E}-02$

$1.85 \mathrm{E}-02$

$2.46 \mathrm{E}-02$

$3.71 \mathrm{E}-02$

$4.30 \mathrm{E}-02$

4.94E-02

7.97E-02

1.52E-02 GSTM1, GSTM2, GSTM3, GSTM4, IDH2, IDH1, RRM2B, MGST1

UGT1A5, ALDH2, UGDH, UGT2B10

ALDH6A1, MUT, ALDH2, ABAT, ACSS2

UGT1A5, UGDH, UGT2B10, DCXR

ACSM3, HMGCS1, ALDH2, ABAT, BDH2

STS, HSD3B1, UGT1A5, SULT2B1, UGT2B10

UGT1A5, PGM1, HK2, UGDH, UGT2B10

GNE, GFPT1, PGM1, HK2, UGDH

STS, HSD3B1, UGT1A5, SULT2B1, UGT2B10

RDH11, UGT1A5, DHRS4L2, UGT2B10, PNPLA4

2.18E-02 PYGL, UGT1A5, PGM1, HK2, UGDH, UGT2B10, PGM2L1

2.69E-02 ALDH6A1, ACADSB, MUT, HMGCS1, ALDH2, ABAT, ACAA1

3.15E-02 ACSM3, HMGCS1, ALDH2, ABAT, BDH2, GAD1

4.38E-02 GSTM1, GSTM2, GSTM3, GSTM4, UGT1A5, MAOB, UGT2B10, MGST1

5.74E-02 UGT1A5, ALDH2, UGDH, UGT2B10

6.63E-02 UGT1A5, UGDH, UGT2B10, DCXR

8.61E-02 ALDH6A1, MUT, ALDH2, ABAT, ACSS2

Fisher's exact test was used to determine whether the proportions of genes falling into each category differs by group. In DAVID annotation system, Fisher's exact test was adopted to measure the gene-enrichment in annotation terms. $\mathrm{P}<0.1$ was used as the cut-off. 

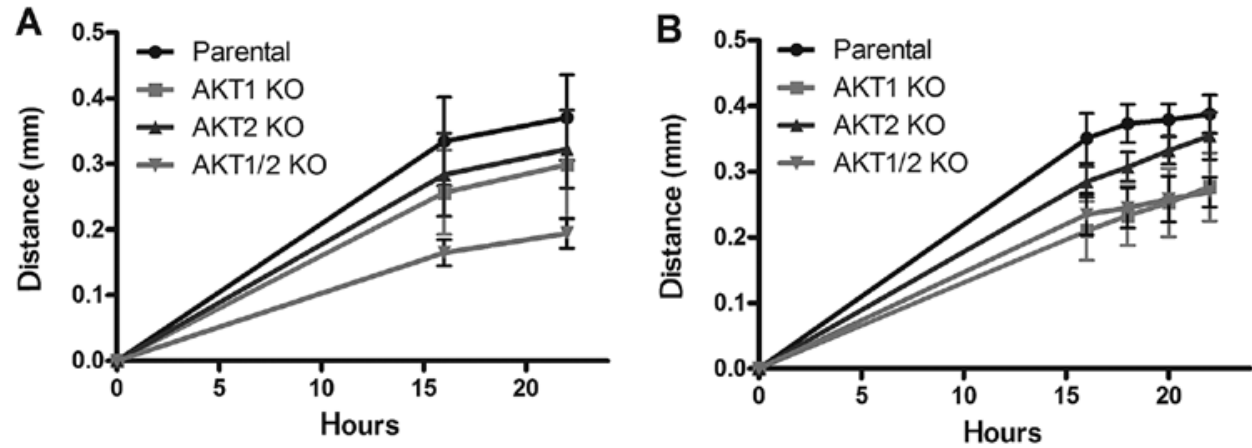

Figure 4. Scratch wound migration assay of DLD-1 and DLD-1 AKT knockout cells. (A) The cell migration under normal conditions. The knockout of $A K T$ showed a clear reduction in cell migration compared to parental. The dual knockout of $A K T 1 / 2$ showed the most prominent effect (P<0.001) at 16 and $22 \mathrm{~h}$, followed by $A K T 1 \mathrm{KO}(\mathrm{P}<0.05)$ and $A K T 2 \mathrm{KO}(\mathrm{P}>0.05)$. (B) Cell migration of cells that were starved for $24 \mathrm{~h}$ before addition of EGF showed that $A K T 1 \mathrm{KO}$ and $A K T 1 / 2 \mathrm{KO}$ have a similar reduction in migration rate compared to parental cells $(\mathrm{P}<0.001$ for both at $16-22 \mathrm{~h})$, followed by $A K T 2 \mathrm{KO}(\mathrm{P}<0.05)$. The error bars represent the standard deviation from at least triplicates.

cells. CD44 and several integrins; ITGA1, ITGA2 and ITGB5 were upregulated whereas CD47, ITGA3, ITGA9, LAMA2, $L A M B 1, S Y D C 1$ and $T N C$ were downregulated. Similar patterns were seen in the $A K T 1$ and $A K T 2 \mathrm{KO}$.

In the Notch-signaling pathway, the $A K T 1 / 2 \mathrm{KO}$ and $A K T 1$ $\mathrm{KO}$ had several genes that were downregulated compared to parental; HES1, MAML2 and JAG1. In the AKT1/2 KO,DTX4 and $K A T 2 B$ were also downregulated. In the $A K T 2 \mathrm{KO}$, there was a similar trend, with the exception of $K A T 2 B$.

Genes involved in the Wnt signaling pathway, were altered in all $A K T$ isoform $\mathrm{KO}$ cells in relation to parental cells. In the $A K T 1 / 2 \mathrm{KO}$, genes that were upregulated include; FZD6, FZD7, MYC, WNT16, DACT1 and TGFB1I1 while AXIN2, $R A C 2$ and $T G F B 3$ were downregulated. In the $A K T 1 \mathrm{KO}$, DACT1, TGFB1I1, TGFB2 and WNT5A were upregulated while LEF1 was downregulated. In $A K T 2 \mathrm{KO}, T G F B 2$ was upregulated while $A X I N 2$ was downregulated.

Genes involved in the p53 pathway, were mostly upregulated in the $A K T 1 / 2 \mathrm{KO}$; CCNE1, CCNE2, CDK6, PMAIP1, RRM2B, SERPINB5 and SESN3, while SKP2 was downregulated. A similar pattern was seen in the $A K T 1 \mathrm{KO}$ compared to parental with SERPINB5 and THBS1 upregulated and SKP2 downregulated. In the $A K T 2 \mathrm{KO}$ SERPINB5 was upregulated while SESN3 was downregulated.

In the metabolic pathways, that were significantly different between the $A K T$ isoform $\mathrm{KO}$ cells and parental cells were the glutathione and drug metabolism pathways (Table II). In the AKT1/2 KO compared to parental, GSTM1, GSTM2, GSTM3, GSTM4, IDH2, IDH1, MGST1, UGT1A5, UGT2B10 were downregulated while $M A O B$ and $R R M 2 B$ were upregulated. In the carbohydrate metabolism (including sucrose, starch and glucose) $P Y G L, P G M 2 L 1$ and $A P P L 1$ were upregulated and UGT1A5, PGM1, HK2, UGDH, UGT2B10,DCXR and $A L D H 2$ were downregulated. Similar patterns were seen in the $A K T 1$ and $A K T 2 \mathrm{KO}$, but not all genes were significantly altered. In the fatty acid metabolism pathway, $A C A A 2$, ALDH7A1, ACSL5 were downregulated in the AKT2 KO. The gene expression of ACAA2 was also downregulated in $A K T 1$ $\mathrm{KO}$, but upregulated in the $A K T 1 / 2 \mathrm{KO}$. The gene expression of $C P T 1 A$ was downregulated in $A K T 1 / 2 \mathrm{KO}$. In the folate cycle, $A L P P$ and $A L P P L 2$ were upregulated, while $S P R$ was downregulated in the $A K T 1 \mathrm{KO}$ and $A K T 1 / 2 \mathrm{KO}$.
Cell migration. The scratch wound healing assay demonstrated a reduced cell migration rate in the $A K T \mathrm{KO}$ cell lines compared to the parental cell line (Fig. 4). In complete media, the $A K T 1 / 2 \mathrm{KO}$ cells demonstrated the most pronounced reduction in migration rate $(\mathrm{P}<0.001)$ at 16 and $22 \mathrm{~h}$, followed by $A K T 1 \mathrm{KO}(\mathrm{P}<0.05)$, and $A K T 2 \mathrm{KO}(\mathrm{P}>0.05)$ (Fig. 4A). Since growth factors influence the migration rate, the cells were also starved for $24 \mathrm{~h}$ before the addition of $15 \mathrm{ng}$ EGF (Fig. 4B). In these settings, all different $A K T \mathrm{KO}$ cells migrated more slowly than the parental cells. The migration rates of the $A K T 1 / 2 \mathrm{KO}$ and $A K T 1 \mathrm{KO}$ were the most reduced compared to the parental cells $(\mathrm{P}<0.001$ for both at $16-22 \mathrm{~h})$, followed by AKT2 KO $(\mathrm{P}<0.05)$.

Metabolic profiling of the polar metabolome. To further explore possible metabolic alterations, the small polar metabolites were analyzed with NMR spectroscopy and evaluated with multivariate modeling in both $A K T \mathrm{KO}$ cell lines and the parental cell line. The differences in the polar metabolome between the parental cell line and the $A K T \mathrm{KO}$ cells were identified using OPLS-DA models and visualized using S-line plots. Moreover, PCA analysis was used to overview the data (Fig. 1). The altered spectral bins, and consequently the annotated metabolites, were tested for significance using ANOVA followed by LSD Tukey's test (Table III). Significant alterations $(\mathrm{P}<0.05)$ were observed in $A K T \mathrm{KO}$ cells and include decreased levels of lactate, alanine and succinic acid as well as increased levels of formate. Moreover, AKT2 $\mathrm{KO}$ and $A K T 1 / 2$ $\mathrm{KO}$ demonstrated a significant increase of glucose metabolism, and $A K T 1 / 2 \mathrm{KO}$ also showed an increase in glutathione and glutamine. The AKT1 KO had reduced levels of the branched amino acids leucine, isoleucine and valine. Additionally, the $A K T 1 \mathrm{KO}$ and $A K T 1 / 2 \mathrm{KO}$ displayed reduced levels of glycine or reduced glycine levels.

\section{Discussion}

The aim of the present study was to elucidate how different pathways involved in cell survival, invasion, metastasis, and resistance to therapy were altered in AKT isoform knockout cell lines compared to their parental cell line. This was done using genome wide expression profiling together with 
Table III. Summary of metabolite changes between AKT knockout cells and parental cells measured with NMR.

\begin{tabular}{|c|c|c|c|c|c|c|c|}
\hline \multirow[b]{2}{*}{ Metabolite ID } & \multirow[b]{2}{*}{ Chemical shift } & \multicolumn{2}{|c|}{$A K T 1 \mathrm{KO}$} & \multicolumn{2}{|c|}{$A K T 2 \mathrm{KO}$} & \multicolumn{2}{|c|}{$A K T 1 / 2 \mathrm{KO}$} \\
\hline & & Alteration & P-value & Alteration & P-value & Alteration & P-value \\
\hline Alanine & 1.47 & - & 0.0005 & $\mathrm{NC}$ & & - & 0.00003 \\
\hline Leucine, isoleucine & 0.96 & $\mathrm{NC}$ & & + & 0.0344 & $\mathrm{NC}$ & \\
\hline Valine & 1.03 & $\mathrm{NC}$ & & + & 0.0151 & $\mathrm{NC}$ & \\
\hline Glycine & 3.55 & $\mathrm{NC}$ & & $\mathrm{NC}$ & & - & 0.0075 \\
\hline Proline & $2.0,3.34$ & - & 0.0045 & - & 0.00004 & - & 0.005 \\
\hline Glucose & $3.24,3.41,3.52,3.88,4.64,5.22$ & $\mathrm{NC}$ & & + & 0.0028 & $\mathrm{NC}$ & \\
\hline Lactate & $1.31,4.10$ & $\mathrm{NC}$ & & - & 0.0008 & - & 0.0082 \\
\hline Glutathione $^{\mathrm{a}}$ & $2.16,2.55,2.97$ & $\mathrm{NC}$ & & & & + & 0.0007 \\
\hline Succinic acid & 2.39 & - & 0.0021 & - & 0.0063 & - & 0.0015 \\
\hline Formic acid (formate) & 8.44 & + & 0.0135 & + & 0.00002 & + & 0.0132 \\
\hline AMP & $5.93,6.13,8.26,8.57$ & - & 0.0346 & - & 0.0263 & $\mathrm{NC}$ & \\
\hline ADP/ATP & $5.39,6.13,8.26,8.52$ & - & 0.0006 & - & 0.0006 & - & 0.0398 \\
\hline
\end{tabular}

Alterations in metabolites between the respective AKT knockout cells compared to parental. No change (NC), increased level (+) and decreased level (-). The P-values were calculated by ANOVA followed by LSD Tukey's test. Only metabolites with P-values $<0.05$ are presented. a'Identity confirmed by spiking of certified reference standard into parental cell line after metabolite extraction.

metabolic profiling, as well as cell migration studies. Results from these analyses were then related to our previous findings regarding DNA repair, radio-resistance, and cancer stem cell marker expression $(12,13)$.

One of the steps in tumor invasion, motility and metastasis involves the cell adhesion molecules (CAMs), which take part in both intracellular (ICM) and extracellular matrix (ECM) interactions of the cancer cells. Loss of cell adhesion will render the cancer cell more motile and invasive, and has been associated with metastatic properties (21). In this study, we demonstrated that genes in the CAM and ECM pathways were significantly affected by the knockout of $A K T 1$ and $A K T 2$ isoforms, and that a result of this could be reduced migration. The majority of the genes in the CAM and ECM pathways were downregulated in the $A K T 1 / 2 \mathrm{KO}$ cell lines compared to parental cells (Table II). This is in line with the results in the cell culture migration assays, where the $A K T$ knockout cell lines, particularly in the $A K T 1 / 2 \mathrm{KO}$, showed a reduction in cell migration rate compared to parental cells (Fig. 3A). This is also in agreement with previous studies, by Ericson et al (22), showing that the $A K T 1 / 2 \mathrm{KO}$ formed fewer liver metastases compared to parental cells in mouse xenografts. Furthermore, the AKT2 KO cells demonstrated an upregulation of genes in the cell adhesion pathway. This could explain the somewhat higher migration rate seen for AKT2 KO cells under EGF treatment (Fig. 3B). Moreover, $A K T 2 \mathrm{KO}$ cells express CD133, as opposed to $A K T 1$ and $A K T 1 / 2 \mathrm{KO}$ cell lines (13). Notably, all the $A K T$ isoform $\mathrm{KO}$ cells had reduced cell migration compared to parental cells, despite their upregulation of CD44, which is associated with increased cell-cell and cell-ECM interactions, migration and metastasis (13).

Pathways that are proposed to be involved in radiation resistance, cell survival and cancer stem cell marker expression are the Notch, Wnt and p53 pathways. The Notch signaling pathway plays an important role in cell survival, embryonic development, proliferation, differentiation and metastasis (23). The Notch pathway has also a role in cancer stem cells and radiation resistance in malignant glioma (21) and breast cancer (24). Furthermore, Notch pathway inhibitors have been shown to increase radiation sensitivity in head and neck cancer and colon cancer xenografts (25). In the present study, we show that $A K T \mathrm{KO}$ caused a reduction in several genes in the Notch signaling pathway (DTX4, HES1, KAT2B, MAML2 and JAG1). This reduction was seen in $A K T 1 / 2 \mathrm{KO}, A K T 1 \mathrm{KO}$ and to some extent in $A K T 2 \mathrm{KO}$, which might explain the increase in radiation sensitivity previously seen $(12,13)$ as well as the effects on cell migration (Fig. 4) in these cell lines compared to parental cells.

Wnt-signaling is another important pathway in colorectal cancer and cancer stem cells with $\beta$-catenin as the key player (26) (Fig. 2). The colon cancer cell line, DLD-1, used in the present study has a mutation in APC which is suggested to make this pathway constantly active. However, despite this mutation, we demonstrated that the $A K T 1 / 2 \mathrm{KO}$ cells have several upregulated genes (FZD6, FZD7, MYC, WNT16, DACT1 and TGFB1I1) as well as others downregulated (AXIN2, RAC2 and TGFB3) in this pathway. This is also in line with Yang et al (27) showing that APC might still phosphorylate $\beta$-catenin despite its mutation. Furthermore, AXIN2 was downregulated in the $A K T 1 \mathrm{KO}, A K T 2 \mathrm{KO}$ and $A K T 1 / 2$ $\mathrm{KO}$ cell lines compared to their parental cell line, which might result in the upregulation of the genes in the Wnt-pathway, subsequently leading to an upregulation of genes like CD44 as shown in a previous study (13).

p53 is a tumor suppressor, which is mutated in numerous cancer forms. The colon cancer cell line DLD-1 has one mutated p53 allele and one p53 silent allele (S241F/SIL) and has no effect on the expression of p21 (28). AKT phosphorylates MDM2, which following phosphorylation, binds to p53 
and causes p53 degradation. In contrast, p53 when active, can inhibit the expression of AKT via PTEN. The $A K T 1 / 2 \mathrm{KO}$ cell line had an increase in the expression of genes involved in the p53 pathway compared to parental cells, however, the regulation of these genes might not be guided by p53 in this case. The genes that were upregulated include PMAIPI (Noxa) which is involved in apoptosis, SERPINB5 and SESN3 involved in inhibition of metastasis and angiogenesis, and $R R M 2 B$ and THSB1, which are involved in DNA repair.

The metabolic pathways are important for cell survival and drug resistance. In this study, we have demonstrated the effects of $A K T$ isoform $\mathrm{KO}$ on metabolism, with an altered expression of genes involved in metabolism as well as metabolic end products. The main sources of energy are glucose and glutamine and alterations in their metabolic pathways effect cell growth. A number of genes involved in the metabolic pathways of glucose and starch were downregulated in the $A K T 1 / 2$ $\mathrm{KO}$ cell line compared to parental cells. These genes are involved in different parts of driving the metabolism of starch and glucose forward (ALDH2, DCXR, HK2, PGM1, UGDH, UGT1A5 and UGT2B10). However, PYGL, which converts stored glycogen into glucose, was upregulated in $A K T 1 \mathrm{KO}$, $A K T 2 \mathrm{KO}$ and $A K T 1 / 2 \mathrm{KO}$. The metabolic profiling experiment by NMR-spectroscopy also demonstrated that lactate was reduced in $A K T 2 \mathrm{KO}$ and $A K T 1 / 2 \mathrm{KO}$ cells and that alanine was reduced in $A K T 1 \mathrm{KO}$ and $A K T 1 / 2 \mathrm{KO}$ cells compared to the parental. This might suggest that the glutamine metabolism and the 'aerobic glycolysis' (i.e. metabolism of glucose to lactate despite presence of oxygen) often seen in cancer cells were reduced (29) (Table III). This would support the theory of AKT-induced glucose metabolism, and that a reduction of AKT can suppress this process. Additionally, the glucose level was increased in the $A K T 2 \mathrm{KO}$ cells compared to parental cells despite the reduced level of lactate, suggesting that these cells may still import glucose even with the lack of AKT2 or at least using a different metabolic pathway compared to parental cells.

The fatty acid synthesis is believed to be promoted by AKT through the activation of enzymes, which convert acetylCoA to fatty acids. Fatty acids are a necessary part of the cell membrane, involved in protein synthesis as well as serve as an essential source of energy and thus proteins involved in the fatty acid synthesis are upregulated in tumor cells (30). AKT regulates the synthesis of proteins and long chain fatty acids via mTOR and fatty acid synthase (FAS). Fatty acids can also be degraded into acetyl-CoA in the mitochondria (via $\beta$-oxidation) and used as an energy source. It is speculated that inhibition of the fatty acid biosynthesis or upregulation of the fatty acid degradation would also impair the tumor cell proliferation (31). In the $A K T 2 \mathrm{KO}$ cells, enzymes involved in the fatty acid degradation, $C P T 1 A, A C A A 2, A L D H 7 A 1$ and $A C S L 5$, were downregulated, which would suggest that the $A K T 2 \mathrm{KO}$ cells have a lack of acetyl-CoA. However, another source of acetyl-CoA is the metabolism of branched amino acids (valine, leucine and isoleucine). As demonstrated in the metabolomic assay, the level of branched amino acids was reduced in the AKTI KO cells. The level of succinic acid, which is a part of the Krebs's cycle, was also reduced in all $A K T$ isoform KO cell lines compared to parental cells. This could be linked to the $I D H 1$ and $I D H 2$ downregulation in the $A K T 1$ and $A K T 1 / 2 \mathrm{KO}$ cells and the downregulation of genes in the fatty acid degradation in $A K T 2 \mathrm{KO}$ cells (32). This would inhibit the Krebs's cycle and cause cells to have a reduced proliferation.

Another important aspect of the metabolic pathways is the resistance to drugs. The inactivation of chemotherapeutic drugs is catalyzed by glutathione S-transferases (GSTs) and these enzymes are believed to play an important role in multiple drug resistance to chemotherapy (33). GSTs also suppress the pro-apoptotic protein ASK1 (apoptosis signal-regulating kinase-1) (34). AKT1/2 KO cells show downregulation of several genes in the glutathione pathway, which suggests that the sensitivity to chemotherapeutics may be enhanced and the number of apoptotic cells increased. The downregulation was, on the other hand, not as prominent in the single $A K T 1 \mathrm{KO}$ and $A K T 2 \mathrm{KO}$ cell lines. Furthermore, the glutathione level was increased in the $A K T 1 / 2 \mathrm{KO}$, which could be a marker for increased oxidative stress (ROS). However, this should be further clarified by studying the level of ROS in the cells, as well as the level of the reduced form of glutathione. The reduced form of glutathione is able to detoxify the cells but the oxidized form cannot. Lactate may also function as a scavenger and reduced levels of lactate in the $A K T 2 \mathrm{KO}$ and $A K T 1 / 2 \mathrm{KO}$ may be part of the higher radiation sensitivity of these cells compared to their parental cells.

The folate cycle is important in cell replication and survival through its involvement in DNA synthesis, DNA repair and DNA methylation (35). ALPPs are elevated in some cancers, including colorectal cancer (36), and have been correlated with folate levels. SPR is involved in the folate cycle as well as in cell proliferation (37). The gene expression of $A L P P$ and $A L P P L 1$ was upregulated in the $A K T 1 \mathrm{KO}$ and $A K T 1 / 2 \mathrm{KO}$ while SPR was downregulated compared to parental. $A K T 1 \mathrm{KO}$ and $A K T 1 / 2 \mathrm{KO}$ also showed decreased levels of glycine that can be correlated with an impaired folate cycle. Additionally, the formate level, which is a metabolite in the folate cycle, was increased in all $A K T$ isoform KO cells, suggesting that the folate cycle was impaired in the $A K T \mathrm{KO}$ cells (38). The impaired folate cycle could be connected to the higher radiation sensitivity in $A K T \mathrm{KO}$ cells compared to parental due to the lack of nucleotides for DNA repair. However, this needs to be further clarified with more extensive metabolite studies.

In summary, this study shows that the dual knockout of both $A K T 1$ and $A K T 2$ in a colorectal cancer cell line (DLD-1) has great impact on several signaling pathways, such as Notch, Wnt, p53 and genes in the cell adhesion and extracellular matrix pathways, in addition to a reduced cell migration rate. The genome analysis and metabolic profiling results show that both $A K T 1$ and $A K T 2$ may change the glucose, starch and sucrose metabolism pathway and that genes in this pathway are mostly downregulated when $A K T 1$ and $A K T 2$ were knocked out. Since lactate and alanine were reduced in $A K T 2$ and $A K T 1 / 2$ $\mathrm{KO}$ cells compared to parental, this further confirms that the aerobic glycolysis and the glutamine catabolism rates were reduced. Genes involved in glutathione and drug metabolism, especially the glutathione S-transferases were downregulated in $A K T 1 / 2 \mathrm{KO}$. This is in line with an observed increase in radiation sensitivity in these cells. The reduction of these pathways confirms that knockout of both $A K T 1$ and $A K T 2$ will attenuate metastasis and tumor cell growth. Notably, genes in 
the Wnt-pathway were upregulated as well as genes that allow progression of the cell cycle, which could oppose this effect. AKT inhibition should therefore be combined with other effectors (drugs, radiation, and inhibitors) to attain the best effect.

\section{Acknowledgements}

The authors would like to acknowledge Hanna Göransson Kultima at the SciLife Laboratory Uppsala, Sweden, for all the help with the gene expression analysis and Ida Erngren for the help with the metabolomic analysis.

\section{References}

1. Pal SK, Reckamp K, Yu H and Figlin RA: Akt inhibitors in clinical development for the treatment of cancer. Expert Opin Investig Drugs 19: 1355-1366, 2010.

2. Cheung M and Testa JR: Diverse mechanisms of AKT pathway activation in human malignancy. Curr Cancer Drug Targets 13 : 234-244, 2013

3. Chandarlapaty S, Sawai A, Scaltriti M, Rodrik-Outmezguine V, Grbovic-Huezo O, Serra V, Majumder PK, Baselga J and Rosen N: AKT inhibition relieves feedback suppression of receptor tyrosine kinase expression and activity. Cancer Cell 19: 58-71, 2011.

4. Hollander MC, Maier CR, Hobbs EA, Ashmore AR, Linnoila RI and Dennis PA: Akt1 deletion prevents lung tumorigenesis by mutant K-ras. Oncogene 30: 1812-1821, 2011.

5. Romano G: The role of the dysfunctional akt-related pathway in cancer: Establishment and maintenance of a malignant cell phenotype, resistance to therapy, and future strategies for drug development. Scientifica (Cairo) 2013: 317186, 2013.

6. Virtakoivu R, Pellinen T, Rantala JK, Perälä M and Ivaska J: Distinct roles of AKT isoforms in regulating $\beta 1$-integrin activity, migration, and invasion in prostate cancer. Mol Biol Cell 23: 3357-3369, 2012.

7. Vander Heiden MG, Cantley LC and Thompson CB: Understanding the Warburg effect: The metabolic requirements of cell proliferation. Science 324: 1029-1033, 2009.

8. Gonzalez E and McGraw TE: The Akt kinases: Isoform specificity in metabolism and cancer. Cell Cycle 8: 2502-2508, 2009.

9. Garofalo RS, Orena SJ, Rafidi K, Torchia AJ, Stock JL, Hildebrandt AL, Coskran T, Black SC, Brees DJ, Wicks JR, et al: Severe diabetes, age-dependent loss of adipose tissue, and mild growth deficiency in mice lacking Akt2/PKB beta. J Clin Invest 112: 197-208, 2003.

10. Katome T, Obata T, Matsushima R, Masuyama N, Cantley LC, Gotoh Y, Kishi K, Shiota H and Ebina Y: Use of RNA interference-mediated gene silencing and adenoviral overexpression to elucidate the roles of AKT/protein kinase B isoforms in insulin actions. J Biol Chem 278: 28312-28323, 2003.

11. Simons A, Orcutt K, Madsen J, Scarbrough P and Spitz D: The role of Akt pathway signaling in glucose metabolism and metabolic oxidative stress. In: Oxidative Stress in Cancer Biology and Therapy. Spitz DR, Dornfeld KJ, Krishnan K and Gius D (eds). Humana Press, New York, pp21-46, 2012.

12. Sahlberg SH, Gustafsson AS, Pendekanti PN, Glimelius B and Stenerlow B: The influence of AKT isoforms on radiation sensitivity and DNA repair in colon cancer cell lines. Tumour Biol 35 3525-3534, 2014.

13. Sahlberg SH, Spiegelberg D, Glimelius B, Stenerlöw B and Nestor M: Evaluation of cancer stem cell markers CD133, CD44, CD24: association with AKT isoforms and radioresistance in colon cancer cells. PLoS One: e94621, 2014.

14. Li C and Wong WH: Model-based analysis of oligonucleotide arrays: Expression index computation and outlier detection. Proc Natl Acad Sci USA 98: 31-36, 2001.

15. Smyth GK: Linear models and empirical bayes methods for assessing differential expression in microarray experiments. Stat Appl Genet Mol Biol 3: Article3, 2004.

16. Benjamini $\mathrm{Y}$ and Hochberg Y: Controlling the false discovery rate: A practical and powerful approach to multiple testing. J R Stat Soc B 57: 289-300, 1995.
17. Liang CC, Park AY and Guan JL: In vitro scratch assay: A convenient and inexpensive method for analysis of cell migration in vitro. Nat Protoc 2: 329-333, 2007.

18. Sumner LW, Amberg A, Barrett D, Beale MH, Beger R, Daykin CA, Fan TW, Fiehn O, Goodacre R, Griffin JL, et al: Proposed minimum reporting standards for chemical analysis Chemical Analysis Working Group (CAWG) Metabolomics Standards Initiative (MSI). Metabolomics 3: 211-221, 2007.

19. Wishart DS, Jewison T, Guo AC, Wilson M, Knox C, Liu Y, Djoumbou Y, Mandal R, Aziat F, Dong E, et al: HMDB 3.0 - The Human Metabolome Database in 2013. Nucleic Acids Res 41 (D1): D801-D807, 2013.

20. Trygg J and Wold S: Orthogonal projections to latent structures (O-PLS). J Chemometr 16: 119-128, 2002.

21. Makrilia N, Kollias A, Manolopoulos L and Syrigos K: Cell adhesion molecules: Role and clinical significance in cancer. Cancer Invest 27: 1023-1037, 2009.

22. Ericson K, Gan C, Cheong I, Rago C, Samuels Y, Velculescu VE, Kinzler KW, Huso DL, Vogelstein B and Papadopoulos N: Genetic inactivation of AKT1, AKT2, and PDPK1 in human colorectal cancer cells clarifies their roles in tumor growth regulation. Proc Natl Acad Sci USA 107: 2598-2603, 2010.

23. Takebe N, Nguyen D and Yang SX: Targeting notch signaling pathway in cancer: Clinical development advances and challenges. Pharmacol Ther 141: 140-149, 2014.

24. Phillips TM, McBride WH and Pajonk F: The response of CD24low/CD $44^{+}$breast cancer-initiating cells to radiation. J Natl Cancer Inst 98: 1777-1785, 2006

25. Liu SK, Bham SA, Fokas E, Beech J, Im J, Cho S, Harris AL and Muschel RJ: Delta-like ligand 4-notch blockade and tumor radiation response. J Natl Cancer Inst 103: 1778-1798, 2011.

26. de Sousa EM, Vermeulen L, Richel D and Medema JP: Targeting Wnt signaling in colon cancer stem cells. Clin Cancer Res 17: 647-653, 2011.

27. Yang J, Zhang W, Evans PM, Chen X, He X and Liu C: Adenomatous polyposis coli (APC) differentially regulates betacatenin phosphorylation and ubiquitination in colon cancer cells. J Biol Chem 281: 17751-17757, 2006.

28. Sur S, Pagliarini R, Bunz F, Rago C, Diaz LA Jr, Kinzler KW, Vogelstein B and Papadopoulos N: A panel of isogenic human cancer cells suggests a therapeutic approach for cancers with inactivated p53. Proc Natl Acad Sci USA 106: 3964-3969, 2009.

29. DeBerardinis RJ, Mancuso A, Daikhin E, Nissim I, Yudkoff M, Wehrli S and Thompson CB: Beyond aerobic glycolysis: Transformed cells can engage in glutamine metabolism that exceeds the requirement for protein and nucleotide synthesis. Proc Natl Acad Sci USA 104: 19345-19350, 2007.

30. Menendez JA and Lupu R: Fatty acid synthase and the lipogenic phenotype in cancer pathogenesis. Nat Rev Cancer 7: 763-777, 2007.

31. Currie E, Schulze A, Zechner R, Walther TC and Farese RV Jr: Cellular fatty acid metabolism and cancer. Cell Metab 18: 153-161, 2013.

32. Oermann EK, Wu J, Guan KL and Xiong Y: Alterations of metabolic genes and metabolites in cancer. Semin Cell Dev Biol 23: 370-380, 2012.

33. Johansson K, Ahlen K, Rinaldi R, Sahlander K, Siritantikorn A and Morgenstern R: Microsomal glutathione transferase 1 in anticancer drug resistance. Carcinogenesis 28: 465-470, 2007.

34. Cho SG, Lee YH, Park HS, Ryoo K, Kang KW, Park J, Eom SJ, Kim MJ, Chang TS, Choi SY, et al: Glutathione S-transferase mu modulates the stress-activated signals by suppressing apoptosis signal-regulating kinase 1. J Biol Chem 276: 12749-12755, 2001.

35. Choi SW and Mason JB: Folate and carcinogenesis: An integrated scheme. J Nutr 130: 129-132, 2000.

36. Harmenberg U, Frodin JE, Ljungdahl-Stahle E, Mellstedt H, Wahren B and Stigbrand T: Significance of alkaline phosphatase isozymes in the monitoring of patients with colorectal carcinoma. Tumour Biol 10: 225-231, 1989.

37. Cho YR, Kim SH, Ko HY, Kim MD, Choi SW and Seo DW: Sepiapterin inhibits cell proliferation and migration of ovarian cancer cells via down-regulation of p70S6K-dependent VEGFR-2 expression. Oncol Rep 26: 861-867, 2011.

38. Lamarre SG, Morrow G, Macmillan L, Brosnan ME and Brosnan JT: Formate: an essential metabolite, a biomarker, or more? Clin Chem Lab Med 51: 571-578, 2013. 